\title{
AMF Otimização de usinas virtuais através de algoritmos evolutivos
}

\author{
Márcio Bürger Mansilha ${ }^{1}$ \\ Felix Alberto Farre ${ }^{2}$
}

\begin{abstract}
Resumo: Com a introdução da geração distribuída (DG) em sistemas elétricos, cirúrgico o conceito de usina virtual (VPP) para gerenciar a integração de pequenas unidades geradoras e lidar efetivamente com a concorrência no mercado de energia. Este documento examina os VPPs em termos de demanda por componentes e sistemas operacionais. $\mathrm{O}$ método de otimização baseado em algoritmos evolutivos é usado. $\mathrm{O}$ VPP proposto tentará fornecer aos consumidores localmente. No entanto, se a energia total produzida pela DG não for suficiente ou muito cara para cobrir as cargas, a energia é comprada da rede, vendida aos consumidores ou armazenada nos dispositivos afetados. A função objetivo é minimizar o custo operacional do VPP, controlando a geração de energia local, bem como seu relacionamento interativo com a rede. As simulações utilizadas pelo algoritmo genético utilizado alcançam um resultado satisfatório. Este trabalho contribui para pesquisadores com diferentes pontos de vista, buscando aplicações mais plausíveis com o VPP.
\end{abstract}

Palavras-chave: Usinas virtuais; resposta de demanda; gerações distribuídas.

\section{Optimization of the virtual power plants through evolutionary algorithms}

Abstract: With the introduction of distributed generation (DG) in electrical systems, the concept of virtual power plant (VPP) has emerged to manage the integration of small generating units and effectively deal with competition in the energy market. This paper examines VPPs in terms of demand for components and operating systems. The optimization method based on evolutionary algorithms is used. The proposed VPP will attempt to supply consumers locally. However, if the total energy produced by DGs is not sufficient, or very expensive to cover the loads, the energy will be purchased from the grid, sold to consumers or stored in the respective devices. The objective function is to minimize the operational cost of VPP by controlling the local power generation, as well as its interactive relation with the network. The simulations show that the genetic algorithm used achieves a satisfactory result. This work contributes that

${ }^{1}$ Doutorando em Engenharia pela Universidade Federal de Santa Maria, com foco em energias renováveis. Mestrado em Engenharia de Produção pela Universidade Federal de Santa Maria . MBA pela Fundação Getúlio Vargas em Finanças Empresariais. Possui graduação em Administração - Comércio Exterior pela Universidade do Vale do Rio dos Sinos

${ }^{2}$ Bacharel e mestre em Engenharia Elétrica pela Universidade Federal de Santa Maria, respectivamente em 1972 e 1976; especialista em instrumentação eletrônica pelo Osaka Prefectural Industrial Research Institute, Japão, em 1975; MSc pela Universidade de Manchester, UMIST, Inglaterra em 1981, PhD em Engenharia Elétrica pela Universidade de Londres, Imperial College, Inglaterra em 1984 e Pós-Doutorado em fontes alternativas de energia na Colorado School of Mines, EUA em 2003. Foi professor visitante na Colorado School of Mines na Divisão de Engenharia, EUA em 2002-2003. Atualmente é professor titular no Departamento de Processamento de Energia Elétrica da Universidade Federal de Santa Maria, Brasil. 
researchers with different points of view look for more plausible applications with VPPs.

Keywords: Virtual power plants; demand response; distributed generations.

\section{Optimización de las centrales virtuales mediante algoritmos evolutivos}

Resumen: Con la introducción de la generación distribuida (DG) en los sistemas eléctricos, surgió el concepto de planta de energía virtual (VPP) para gestionar la integración de pequeñas unidades generadoras y lidiar efectivamente con la competencia en el mercado energético. Este documento examina los VPP en términos de demanda de componentes y sistemas operativos. Se utiliza el método de optimización basado en algoritmos evolutivos. El VPP propuesto intentará suministrar a los consumidores localmente. Sin embargo, si la energía total producida por las DG no es suficiente o muy costosa para cubrir las cargas, la energía se comprará de la red, se venderá a los consumidores o se almacenará en los dispositivos respectivos. La función objetivo es minimizar el costo operativo de VPP controlando la generación de energía local, así como su relación interactiva con la red. Las simulaciones muestran que el algoritmo genético utilizado logra un resultado satisfactorio. Este trabajo contribuye a que los investigadores con diferentes puntos de vista busquen aplicaciones más plausibles con VPP.

Palabras clave: Plantas de energía virtual; respuesta a la demanda; generaciones distribuidas.

\section{INTRODUÇÃO}

Assegurar que a demanda de energia seja atendida sem interrupções é um desafio. Quando a energia é produzida unicamente pelas centrais elétricas convencionais a confiabilidade é facilmente tratada. O problema torna-se premente quando são envolvidas fontes de energia renováveis (ROBU et al., 2016). A pequena capacidade instalada, intermitência, incerteza e outras características comuns destas fontes são um obstáculo à sua entrada e participação no mercado de energia e serviços auxiliares (JU et al., 2016; SHAYEGAN-RAD; BADRI; ZANGENEH, 2017).

$\mathrm{O}$ conceito de integração de pequenas unidades geradoras no sistema de energia tem atraído muito a atenção dos engenheiros e pesauisadores nos últimos anos. Além disso, a geração distribuída (DG) desempenha um papel importante no reforço das principais usinas geradoras para satisfazer a crescente demanda por energia (OTHMAN; HEGAZY; ABDELAZIZ, 2015). Com o aumento da inserção das DGs foram detectados dois grandes problemas (NEZAMABADI; SETAYESH NAZAR, 2016): a) estes elementos não são visíveis para o operador de sistema para utilizá-los de forma eficiente e b) não podem participar nos mercados 
de energia e serviços auxiliares para obter lucros. Ainda assim passarão a existir oportunidades para as VPPs e micro-redes (ASMUS, 2010).

Os objetivos deste artigo visam apresentar as definições, componentes e estruturas das VPP's, analisar um estudo de caso de VPP composta por micro e minigeradores de energia e aplicar o modelo de otimização no ambiente software MATLAB para minimizar os custos totais da VPP.

\section{A. Conceito atual de usina elétrica virtual (VPP)}

O conceito de VPP tem várias definições, mas todos concordam com o fato de que a VPP é uma agregação de unidades DG de diferentes tecnologias (OTHMAN; HEGAZY; ABDELAZIZ, 2015) e proprietários. Portanto, uma VPP é composta de um número de DGs, incluindo usinas elétricas convencionais e unidades geradoras intermitentes, juntamente com possíveis cargas flexíveis e unidades de armazenamento (GHAVIDEL et al., 2016).

A existência da VPP foi proposta como uma nova tecnologia para os recursos energéticos distribuídos (DERs) no mercado de energia. Sem modificar o método de conexão da rede do DERs, a VPP integra diferentes tipos de DERs, como fontes de energia distribuídas, armazenamento de energia, sistemas e cargas controláveis com a utilização de tecnologias avançadas para controle, cálculo e comunicação (JU et al., 2016; ZAPATA RIVEROS et al., 2015). A VPP gerencia recursos agregados dos sistemas de distribuição, que, inteligentemente, utilizam DERs como coalizão de tecnologias heterogéneas para poder negociar a energia no mercado livre de energia (RAHMANI-DABBAGH; SHEIKH-EL-ESLAMI, 2016; SHABANZADEH; SHEIKH-EL-ESLAMI; HAGHIFAM, 2016). A VPP é conhecida como uma agregação técnica onde um agregador agrupa a flexibilidade da geração distribuída e da resposta à demanda, oferecendo os recursos coletivos para o mercado de energia (MACDOUGALL et al., 2016). A VPP representa a "Internet da energia", aproveitando as redes existentes para adaptar os serviços de fornecimento e demanda de eletricidade a um cliente. Com isto, maximiza-se o valor tanto para o usuário final quanto para a distribuição através de inovações de software (ASMUS, 2010).

A VPP consiste de três componentes principais: recursos energéticos distribuídos, sistemas de armazenamento de energia e tecnologias de informação (OTHMAN; HEGAZY; ABDELAZIZ, 2015).

\section{Recursos energéticos distribuídos (DERs)}


DER podem ser geradores distribuídos ou controláveis pelas cargas ligadas à rede. As DGs das instalações do VPP podem ser classificadas de acordo com:

a) Tipo de fonte de energia primária:

b) Capacidade das unidades DG:

c) Propriedade das unidades DG:

d) Natureza operacional das DG.

2. $\quad$ Sistemas de armazenamento de energia (ESS)

3. Sistemas de informação e comunicação

O conhecimento da disponibilidade em tempo real das condições energéticas do sistema é vital para garantir a prestação de um serviço oferecido, bem como para desenvolver estratégias de negociação economicamente ótimas. Para tal, a VPP deve estimar uma capacidade de energia adequada a partir das previsões de sua flexibilidade de recursos de DR juntamente com estimativas esperadas em tempo real do perfil da energia. O VPP pode participar no mercado de energia como "day-ahead" e "intra-day”, bem como, em serviços, tais como frequência primária e suporte de controle para a tensão ou o balanceamento de energia em tempo real (OTHMAN; HEGAZY; ABDELAZIZ, 2015).

O sistema de gestão de energia (EMS) está associado com política, mercado de eletricidade, carga / previsão de preço / DER, clientes, utilitário, cargas e DERs em uma microgrid. O EMS recebe os dados de previsão dos recursos de energia e de carga, informações sobre preferências de clientes, informações de mercado e de energia. Com esta informações, o EMS determina os melhores controles disponíveis no fluxo de energia, compras de energia elétrica, despacho de carga e pré-formação do DER (NOSRATABADI; HOOSHMAND; GHOLIPOUR, 2017).

O VPP não só procura participar no mercado de energia “day-ahead”, mas também busca aumentar o seu lucro através da participação no mercado.

No artigo (SHAYEGAN-RAD; BADRI; ZANGENEH, 2017), a função objetivo de maximizar o lucro esperado da VPP é calculado na equação (1): 
$\operatorname{Lucro}(h)=\sum_{h}-C_{O}^{S D G}(h)+R^{E V}(h)+R_{E}^{D A}(h)+R_{R R}^{D A}(h)$

Onde o primeiro termo da função lucro naado na equação (1): representa a operação da geração distribuída síncrona (SDG) para gerar energia. O segundo termo representa a receita VPP ao utilizar veículos elétricos (EVs). O terceiro e quarto termos são receitas da venda de energia e fornecimento do mercado de reserva de regulamentação (RR) para o mercado dayahead, respectivamente.

As principais contribuições deste artigo (SHAYEGAN-RAD; BADRI; ZANGENEH, 2017) são:

- $\quad$ Propõe um modelo que incorpora os três tipos de recursos energéticos: as pequenas centrais convencionais (SDG), as fontes de geração intermitentes e as instalações de armazenamento nos mercados de reserva diurnos e regulatórios.

- $\quad$ O VPP fornecerá RR baseado no pedido de regulação up / down do mercado regulatório com preço estocástico e probabilidade de chamada.

- O VPP estabelecerá formas de contratos para incentivo de veículos elétricos (EVs) para usar veículo-to-grid (V2G) como DER; o custo da penalidade das emissões de CO2 estará incluído no custo de operação da SDG.

- $\quad$ Propõe um método de estimativa de $2 \mathrm{~m} \mathrm{p} 1$ ponto (PEM) como sendo uma técnica eficiente e robusta para modelar incertezas; esta técnica é usada para modelos nãodeterminados de parâmetros incluindo a energia diurna e os preços de mercado de RR, a regulação de probabilidades de chamada para cima e para baixo, a geração de WPP e o número de EVs de entrada (saída) para (a partir) do estacionamento.

\section{B. Algoritmo Genético (GA)}

Inspirado na maneira como o darwinismo explica o processo de evolução das espécies. $\mathrm{O}$ GA cria uma população de possíveis respostas para o problema a ser tratado para depois submetê-la ao processo de evolução (LUCAS, 2002). As vantagens dos GA sob outras técnicas de otimização são: os GA não utilizam métodos de busca totalmente aleatórios; os GA não são afetados por descontinuidades na função ou em suas derivadas. Isto faz com que os GA sejam adequados para funções com descontinuidades, ou para funções com as quais não se podem 
calcular derivadas; os GA são capazes de lidar com funções discretas e contínuas, podendo inclusive trabalhar com funções mistas; os GA são apropriados para resolver problemas de busca com espaços de busca grandes demais para serem resolvidos por técnicas de otimização tradicionais (PINHO et al., 2013).

\section{Método}

Do ponto de vista dos procedimentos técnicos utilizados destaca-se: a) pesquisa bibliográfica utilizando-se material publicado em livros e artigos científicos; b) estudo de um caso que permite conhecimento do objeto de estudo. A natureza desta pesquisa é aplicada, buscando conhecimentos para aplicação na prática do gerenciamento de VPPs e dirigida à solução de problemas específicos. A forma de abordagem é quantitativa utilizando modelagem matemática, simulação e a avaliação da operação de cada componente de uma VPP. O desenvolvimento desta pesquisa classifica as VPP's quanto: à natureza, à forma de abordagem, aos objetivos e procedimentos técnicos.

O modelo de gerenciamento de energia na VPP inclui DGs renováveis e convencionais. A função objetivo é minimizar o custo operacional da VPP pelo controle da geração de energia local, bem como, sua relação interativa com a rede. Foram utilizadas como base nesta pesquisa a função objetivo (KASAEI; GANDOMKAR; NIKOUKAR, 2017) e utilizou-se o sistema completo de dados de entrada para a simulação (MOGHADDAM et al., 2011).

A VPP proposta tentará abastecer os consumidores localmente. No entanto, se a energia total produzida por DGs não for suficiente, ou for muito dispendiosa para cobrir as cargas, a energia será comprada da rede e vendida aos consumidores ou armazenada nos dispositivos respectivos.

O custo objetivo consiste no poder de troca entre o VPP e a rede, no custo do combustível para as DGs e no custo de inicialização ou desligamento das fontes de energia usadas no VPP. A função proposta é indicada na forma das equações (2), (3), (4) e (5). 


\section{A. Função Objetivo}

Min $f=\sum_{t=1}^{T}$ Custo

$$
\left[\sum_{t=1}^{T} \text { Custo }=\sum_{t=1}^{T}\left(\begin{array}{c}
P_{\text {Rede }}(t) \times C_{\text {Rede }}(t) \\
+U_{W T}(t) \times P_{W T}(t) \times C_{W T}(t) \\
+U_{P V}(t) \times P_{P V}(t) \times C_{P V}(t) \\
+U_{F C}(t) \times P_{F C}(t) \times C_{F C}(t) \\
+U_{M T}(t) \times P_{M T}(t) \times C_{M T}(t) \\
+\sum_{j=1}^{N_{S}} U_{j}(t) \times P_{S J}(t) \times C_{j}(t) \\
+\sum_{i=1}^{N_{g}} S_{G i}\left|U_{i}(t)-U_{i}(t-1)\right| \\
+\sum_{j=1}^{N_{S}} S_{S j}\left|U_{j}(t)-U_{j}(t-1)\right| \\
-\Delta P(t) \times C_{\Delta P}(t)
\end{array}\right)\right.
$$

$\sum_{t=1}^{T} \Delta P(t)=\sum_{t=1}^{T}\left(P_{\text {perdas }}(t)-P_{\text {novas perdas }(t)}\right)$

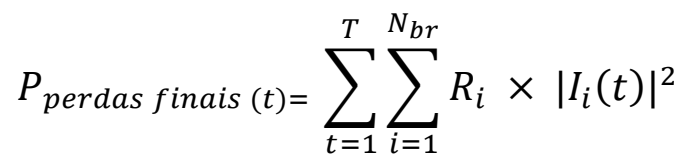

onde:

$\mathrm{C}_{\mathrm{WT}}(\mathrm{t})$ - Custo turbina eólica na hora $\mathrm{t}$;

$\mathrm{C}_{\mathrm{PV}}(\mathrm{t})$ - Custo fotovoltaico na hora $\mathrm{t}$;

$\mathrm{C}_{\mathrm{FC}}(\mathrm{t})$ - Custo célula combustível na hora t;

$\mathrm{C}_{\mathrm{MT}}(\mathrm{t})$ - Custo microturbina na hora $\mathrm{t}$; 
$\mathrm{C}_{\mathrm{j}}(\mathrm{t})$ - Custo dispositivo armazenamento na hora $\mathrm{t}$;

$\mathrm{S}_{\mathrm{GI}}(\mathrm{t})$ - Custos de inicialização;

$\mathrm{S}_{\mathrm{sj}}(\mathrm{t})$ - Custos de desligamento para i DG e j armazenamento;

$P_{\text {Rede }}(t)$ - Potência ativa que é comprada (vendida) de (para) a concessionária de energia no momento t;

$\mathrm{C}_{\text {Rede }}(\mathrm{t})$ - Oferta $(\mathrm{R} \$ / \mathrm{kWh})$ da distribuidora no tempo $\mathrm{t}$;

$\mathrm{N}_{\mathrm{G}}, \mathrm{N}_{\mathrm{s}}$ - Representam o número total de unidades de geração e armazenamento, respectivamente;

$U_{W T}(t), U_{P V}(t), U_{F C}(t), U_{M T}(t), U_{j}(t)$ - Representa os estados ON ou OFF de todas as unidades na hora t do dia;

$\Delta \mathrm{P}(\mathrm{t}), \mathrm{C}_{\Delta \mathrm{P}}(\mathrm{t})$ - Representam a diferença entre as perdas originais e as novas perdas dos alimentadores e o custo na hora t;

$R_{i}, I_{i}$ - Representam a resistência e a corrente reais do $i$-ésimo ramo.

\section{B. Restrições}

1. Equilíbrio de potência em cada período de tempo t:

$\sum_{t=1}^{T}\left(\begin{array}{c}P_{\text {Rede }}(t)+P_{W T}(t) \\ +P_{P V}(t)+P_{F C}(t) \\ +P_{\text {Descarga da bateria }}(t)\end{array}\right)=\sum_{t=1}^{T}\left(\begin{array}{c}\operatorname{Carga}(t) \\ +P_{\text {Cargada Bateria }}(t) \\ +P_{\text {perda }}(t)\end{array}\right)$

2. Limitação de geração da turbina eólica em cada período de tempo t:

$P_{W T \min }(t) \leq P_{W T}(t) \leq P_{W T \max }(t) ; t=1, \ldots, T$

3. Limitação da geração fotovoltaica em cada período de tempo t:

$P_{P V \min }(t) \leq P_{P V}(t) \leq P_{P V \max }(t) ; t=1, \ldots, T$

4. Limitação de células a combustível em cada período de tempo t:

$P_{F C \min }(t) \leq P_{F C}(t) \leq P_{F C \max }(t) ; t=1, \ldots, T$ 
5. Limitação de microturbina em cada período de tempo t:

$P_{M T \min }(t) \leq P_{M T}(t) \leq P_{M T \max }(t) ; t=1, \ldots, T$

6. Limitação de concessionária em cada período de tempo t:

$P_{\text {Redemin }}(t) \leq P_{\text {Rede }}(t) \leq P_{\text {REDEmax }}(t) ; t=1, \ldots, T$

7. Limitação do armazenamento da bateria em cada período de tempo t:

$P_{\text {Sjmin }}(t) \leq P_{S j}(t) \leq P_{\text {Sjmax }}(t) ; t=1, \ldots, T$

Devido à restrição para a carga e à taxa de descarga dos dispositivos de armazenamento durante cada período de tempo, a seguinte equação e restrições podem ser consideradas:

$$
\begin{aligned}
& W_{\text {ess }}(t)=W_{\text {ess }}(t-1)+\eta_{\text {Carregar }} P_{\text {Carregar }} \Delta t-\frac{1}{\eta_{\text {Descarregar }}} P_{\text {Descarregar }} \Delta t \\
& W_{\text {essmin }}(t) \leq W_{\text {ess }}(t) \leq W_{\text {essmax }}(t) ; t=1, \ldots, T \\
& P_{\text {Carregar }}(t) \leq P_{\text {Carregarmax }}(t) ; t=1, \ldots, T ; X \in\{0,1\} \\
& P_{\text {Descarregar }}(t) \leq P_{\text {descarregarmax }}(t) ; t=1, \ldots, T ; Y \in\{0,1\}
\end{aligned}
$$

A bateria não pode carregar e descarregar no mesmo tempo, então:

$X(t)+Y(t) \leq 1 ; t=1, \ldots, 24 ; X$ e $Y \in\{0,1\}$

onde: 
$\mathrm{W}_{\mathrm{ess}}, \mathrm{W}_{\mathrm{ess}}(\mathrm{t}-1)$ - são as quantidades de armazenamento de energia dentro da bateria às horas $\mathrm{t}$ e $\mathrm{t}-1$

$\mathrm{P}_{\text {Carregar }}, \mathrm{P}_{\text {Descarregar }}$ - são as taxas permitidas de carga e descarga através de um período de tempo definido $(\Delta \mathrm{t}=1 \mathrm{~h})$;

$\eta_{\text {Carregar }}, \eta_{\text {Descarregar }}$ - são a eficiência da carga e descarga.

C. Coleta de dados para simulação

O diagrama único do sistema que foi utilizado para iniciar a simulação é mostrado na Figura 1.

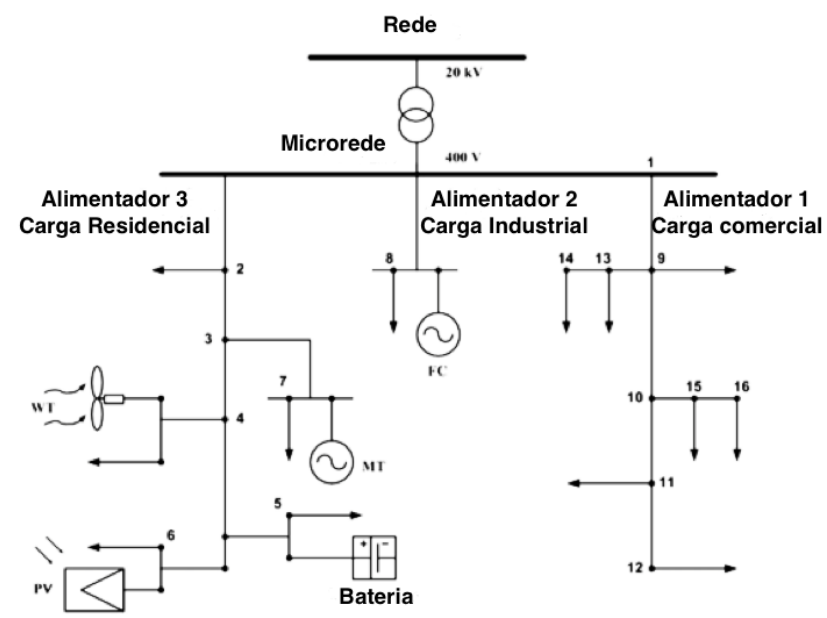

Figura 1 - Diagrama do sistema do estudo de caso

Fonte: adaptado [16].

A Tabela 1 apresenta o preço real no mercado considerado para cada hora do dia. A curva de carga diária da micro-rede para um dia típico, compreendendo uma área residencial, uma área industrial e uma área comercial com consumidores comerciais leves equivalentes a uma demanda total de energia de 1695 kWh para o dia. E a geração máxima de energia obtida de RESs também é estimada para um dia à frente, usando um modelo de previsão de (MOGHADDAM et al., 2011). 
TABELA I

PREÇO DA ENERGIA

\begin{tabular}{lcccc}
\hline \hline Hora & $\begin{array}{c}\text { Preço } \\
(\$ / \mathrm{kWh})\end{array}$ & $\begin{array}{c}\text { Carga } \\
(\mathrm{kW})\end{array}$ & $\begin{array}{c}\text { WT } \\
(\mathrm{kW} / \mathrm{kWinstal})\end{array}$ & $\begin{array}{c}\text { PV } \\
(\mathrm{kW} / \mathrm{kWinstal})\end{array}$ \\
\hline 1 & 0,23 & 52 & 0,119 & 0 \\
2 & 0,19 & 50 & 0,119 & 0 \\
3 & 0,14 & 50 & 0,119 & 0 \\
4 & 0,12 & 51 & 0,119 & 0 \\
5 & 0,12 & 56 & 0,119 & 0 \\
6 & 0,20 & 63 & 0,061 & 0 \\
7 & 0,23 & 70 & 0,119 & 0 \\
8 & 0,38 & 75 & 0,087 & 0,008 \\
9 & 1,50 & 76 & 0,119 & 0,150 \\
10 & 4,00 & 80 & 0,206 & 0,301 \\
11 & 4,00 & 78 & 0,585 & 0,418 \\
12 & 4,00 & 74 & 0,694 & 0,478 \\
13 & 1,50 & 72 & 0,26 & 0,956 \\
14 & 4,00 & 72 & 0,158 & 0,842 \\
15 & 2,00 & 76 & 0,119 & 0,315 \\
16 & 1,95 & 80 & 0,087 & 0,169 \\
17 & 0,60 & 85 & 0,119 & 0,022 \\
18 & 0,41 & 88 & 0,119 & 0 \\
19 & 0,35 & 90 & 0,0868 & 0 \\
20 & 0,43 & 87 & 0,119 & 0 \\
21 & 1,17 & 78 & 0,0867 & 0 \\
22 & 0,54 & 71 & 0,0867 & 0 \\
23 & 0,30 & 65 & 0,061 & 0,041 \\
\hline
\end{tabular}

Tabela 1 - Preço da energia no mercado em tempo real, carga diária do sistema e previsão de geração de WT e PV. Fonte: adaptado de Moghaddam et al (2011). 
Os limites mínimo e máximo de produção de energia das DGs são apresentados na Tabela 2. Os coeficientes de oferta em centavos da unidade monetária por quilowatts-hora (kWh). $\mathrm{Na}$ mesma tabela 2, constam os custos de startup / shutdown, quando aplicáveis.

\section{TABELA II}

\section{LIMITES E OFERTAS PREÇOS DE ENERGIA}

\begin{tabular}{|c|c|c|c|c|c|}
\hline ID & Tipo & $\begin{array}{l}\text { Potência } \\
\text { Mínima (kW) }\end{array}$ & $\begin{array}{l}\text { Potência } \\
\text { Máxima (kW) }\end{array}$ & $\begin{array}{l}\text { Preço } \\
(\$ / k W h)\end{array}$ & $\begin{array}{l}\text { Custo startup / } \\
\text { shutdown }\end{array}$ \\
\hline 1 & MT & 6 & 30 & 0,457 & 0,96 \\
\hline 2 & $\mathrm{FC}$ & 3 & 30 & 0,294 & 1,65 \\
\hline 3 & PV & 0 & 25 & 2,584 & 0 \\
\hline 4 & WT & 0 & 15 & 1,073 & 0 \\
\hline 5 & Bateria & -30 & 30 & 0,380 & 0 \\
\hline 6 & Rede & -30 & 30 & - & - \\
\hline
\end{tabular}

Tabela 2 - Limites e ofertas de preços das RESs e da concessionária de energia.

Fonte: Baziar e Kavousi-Fard (2013).

\section{Parâmetros usados no Matlab}

Para simulação no Matlab foi utilizado a Optimization Tool para uma população com 200 indivíduos. A Função Fitness do tipo ranking escala as pontuações com base na classificação de cada indivíduo em vez de sua pontuação. A classificação de um indivíduo é sua posição nas pontuações classificadas. A classificação do indivíduo mais apto é 1, o próximo mais apto é 2 e assim por diante.

Seleção: a estocástica uniforme estabelece uma linha na qual cada o pai corresponde a uma seção da linha de comprimento proporcional à sua expectativa. Reprodução: Elitismo (0,05*tamanho da população), o número de indivíduos com garantia de sobrevivência até a próxima geração. A fração de cruzamento: 0,8 (a fração da próxima geração produzida pelo crossover). Função crossover: dependente de restrição de dispersão. Mutação: Gaussiana (adiciona um número aleatório a cada entrada de vetor de um indivíduo. Esse número aleatório é retirado de uma distribuição gaussiana centrada em zero). Critérios de Parada para 100 gerações 
estabelecendo-se como número máximo de iterações para o algoritmo genético executar. Limite de tempo de parada: se não houver melhoria no valor de adequação por um intervalo de tempo, o algoritmo é interrompido. Tolerância de função: se a alteração média no valor da função de adequação sobre gerações de stall for menor que a tolerância de função, o algoritmo será interrompido.

\section{DisCUSSÃo E RESULTADOS}

Os dados usados nas simulações foram retirados dos artigos de (KASAEI; GANDOMKAR; NIKOUKAR, 2017), (MOGHADDAM et al., 2011) e (BAZIAR; KAVOUSIFARD, 2013). A função objetivo e as restrições foram modeladas no software MATLAB para validação da técnica da otimização. Foi simulado um cenário para teste onde os DGs podem alternar-se entre modos ON / OFF com uma carga inicial da bateria igual a zero. Isso significa que a quantidade de descargas da bateria é limitada pela quantidade de energia que é carregada nas últimas horas. A Tabela 3 apresenta os resultados do despacho econômico de energia, de acordo com (BAZIAR; KAVOUSI-FARD, 2013).

\section{TABELA III}

\section{DESPACHO ECONÔMICO}

\begin{tabular}{crrrrrr}
\hline \hline T (h) & Rede & WT & PV & FC & MT & Bateria \\
\hline 1 & 30 & 1,79 & 0 & 30 & 20,21 & -30 \\
2 & 30 & 1,79 & 0 & 30 & 18,21 & -30 \\
3 & 30 & 1,79 & 0 & 30 & 18,21 & -30 \\
4 & 30 & 1,79 & 0 & 30 & 19,21 & -30 \\
5 & 30 & 1,79 & 0 & 30 & 24,21 & -30 \\
6 & 30 & 0,92 & 0 & 30 & 30 & $-27,91$ \\
7 & 30 & 1,79 & 0 & 30 & 30 & $-21,78$ \\
8 & 30 & 1,31 & 0,2 & 30 & 30 & $-16,50$ \\
9 & $-19,54$ & 1,79 & 3,75 & 30 & 30 & 30 \\
10 & $-20,62$ & 3,09 & 7,522 & 30 & 30 & 30
\end{tabular}




\begin{tabular}{rrrrrrr}
11 & -30 & 8,78 & 10,45 & 30 & 30 & 28,77 \\
12 & -30 & 10,41 & 11,95 & 30 & 30 & 21,64 \\
13 & -30 & 3,92 & 23,9 & 30 & 30 & 14,18 \\
14 & -30 & 2,37 & 21,05 & 30 & 30 & 18,58 \\
15 & $-23,66$ & 1,79 & 7,87 & 30 & 30 & 30 \\
16 & $-15,53$ & 1,30 & 4,22 & 30 & 30 & 30 \\
17 & 30 & 1,79 & 0,55 & 30 & 30 & $-7,33$ \\
18 & 30 & 1,79 & 0 & 30 & 30 & $-3,78$ \\
19 & 30 & 1,30 & 0 & 30 & 30 & $-1,30$ \\
20 & 30 & 1,79 & 0 & 30 & 30 & $-4,78$ \\
21 & $-13,30$ & 1,30 & 0 & 30 & 30 & 30 \\
22 & 9,47 & 1,30 & 0 & 30 & 30 & 0,23 \\
23 & 30 & 0,92 & 0 & 30 & 6 & $-1,91$ \\
24 & 30 & 0,62 & 0 & 30 & 0 & $-4,61$ \\
\hline
\end{tabular}

Tabela 3: Despacho econômico.

Fonte: Baziar e Kavousi-Fard, 2013.

Constata-se que a bateria é carregada nas primeiras horas do dia (horas 1 e 8), mesmo comprando energia de uma microturbina (MT). Esse evento indica que para reduzir a geração de energia elétrica em horários posteriores é mais econômico carregar a bateria com uma MT.

Um segundo cenário foi simulado onde as DGs podem alternar entre modos ON / OFF, não considerando a descarga da bateria. A Tabela 5 mostra os resultados da comparação de desempenho dos algoritmos. Assim, as simulações demonstram que o algoritmo genético utilizado no software Matlab atende as expectativas atingindo uma solução melhor que o algoritmo utilizado por (KASAEI; GANDOMKAR; NIKOUKAR, 2017).

\section{TABELA IV}

COMPARAÇÃO DE CUSTOS TOTAIS

\begin{tabular}{llll}
\hline \hline Tipo & Melhor Solução & Pior Solução & Média \\
\hline GA [15] & 334,8694 & 345,0211 & 336,2912
\end{tabular}


GA

Tabela 4: Comparação de custos totais.

Fonte: Kasei, Gandomkar e Nikoukar (2017).

\section{CONCLUSÃO}

O objetivo deste artigo é o de apresentar as definições, componentes e estrutura das VPP's. Estes fatores são analisados num estudo de caso de uma VPP composta por micro e mini geradores de energia. Neste estudo foi aplicado o modelo de otimização no ambiente software MATLAB para minimizar os custos totais da VPP.

Para o estudo de caso, o primeiro cenário proposto foi o de que a VPP tentaria abastecer seus consumidores localmente. Se a energia total produzida pelas DGs não fosse suficiente, ou fosse muito dispendiosa para cobrir as cargas, a energia seria comprada da rede e vendida aos consumidores, ou ainda, armazenada nos dispositivos respectivos como demonstrado na tabela 5 . Constatou-se que, a bateria foi carregada nas primeiras horas de dia (horas 1 e 8), mesmo comprando energia microturbina (MT). Esse evento indica que para reduzir a geração de energia elétrica em horários posteriores, é mais econômico carregar a bateria com uma MT.

As simulações propostas neste artigo demonstram que o algoritmo genético utilizado no software MATLAB atende as expectativas atingindo uma solução melhor para minimizar os custos totais da VPP de $\$ 330,6707$ versus $\$ 334,8694$ que o algoritmo utilizado por (KASAEI; GANDOMKAR; NIKOUKAR, 2017).

Simulou-se um segundo cenário onde as DGs podem alternar entre modos ON / OFF, não considerando a descarga da bateria conforme apresentado na tabela 7. O custo objetivo consiste no poder de troca entre o VPP e a rede, o custo do combustível para as DGs e o custo de inicialização ou desligamento das fontes de energia usadas no VPP.

Este trabalho facilita aos pesquisadores com diferentes pontos de vista a busca de novas possíveis aplicações das VPPs.

\section{REFERÊNCIAS}

ASMUS, P. Microgrids, Virtual Power Plants and Our Distributed Energy Future. Electricity Journal, v. 23, n. 10, p. 72-82, 2010.

BAZIAR, A.; KAVOUSI-FARD, A. Considering uncertainty in the optimal energy management 
of renewable micro-grids including storage devices. Renewable Energy, v. 59, p. 158-166, 2013.

GHAVIDEL, S. et al. A review on the virtual power plant: Components and operation systems. 2016 IEEE International Conference on Power System Technology, POWERCON 2016, p. $1-6,2016$.

JU, L. et al. A bi-level stochastic scheduling optimization model for a virtual power plant connected to a wind-photovoltaic-energy storage system considering the uncertainty and demand response. Applied Energy, v. 171, p. 184-199, 2016.

KASAEI, M. J.; GANDOMKAR, M.; NIKOUKAR, J. Optimal management of renewable energy sources by virtual power plant. Renewable Energy, v. 114, p. 1180-1188, 2017.

LUCAS, C. D. Algoritmos Genéticos: uma Introdução. Instituto de Informática, v. Março, p. 48, 2002.

MACDOUGALL, P. et al. Applying machine learning techniques for forecasting flexibility of virtual power plants. 2016 IEEE Electrical Power and Energy Conference, EPEC 2016. Anais...TNO Monitoring and Control Services, The Hague, Netherlands: Institute of Electrical and Electronics Engineers Inc., 2016. Disponível em: $<$ https://www.scopus.com/inward/record.uri?eid=2-s2.0-

85010589160\&doi=10.1109\%2FEPEC. $2016.7771738 \&$ partnerID=40\&md5=bd5a2bd1b98dfbaa 65c912b62946f3c5>.

MOGHADDAM, A. A. et al. Multi-objective operation management of a renewable MG (microgrid) with back-up micro-turbine/fuel cell/battery hybrid power source. Energy, v. 36, n. 11, p. 6490-6507, 2011.

NEZAMABADI, H.; SETAYESH NAZAR, M. Arbitrage strategy of virtual power plants in energy, spinning reserve and reactive power markets. IET Generation, Transmission and Distribution, v. 10, n. 3, p. 750-763, 2016.

NOSRATABADI, S. M.; HOOSHMAND, R. A.; GHOLIPOUR, E. A comprehensive review on microgrid and virtual power plant concepts employed for distributed energy resources scheduling in power systems. Renewable and Sustainable Energy Reviews, v. 67, p. 341-363, 2017.

OTHMAN, M. M.; HEGAZY, Y. G.; ABDELAZIZ, A. Y. A Review of virtual power plant definitions, components, framework and optimization. International Electrical Engineering Journal, v. 6, n. 9, p. 2010-2024, 2015.

PINHO, A. F. DE et al. Algoritmos Genéticos: Fundamentos e Aplicações. [s.l: s.n.].

RAHMANI-DABBAGH, S.; SHEIKH-EL-ESLAMI, M. K. A profit sharing scheme for distributed energy resources integrated into a virtual power plant. Applied Energy, v. 184, p. 313-328, 2016. 
ROBU, V. et al. Rewarding cooperative virtual power plant formation using scoring rules. Energy, v. 117, p. 19-28, 2016.

SHABANZADEH, M.; SHEIKH-EL-ESLAMI, M. K.; HAGHIFAM, M. R. A medium-term coalition-forming model of heterogeneous DERs for a commercial virtual power plant. Applied Energy, v. 169, p. 663-681, 2016.

SHAYEGAN-RAD, A.; BADRI, A.; ZANGENEH, A. Day-ahead scheduling of virtual power plant in joint energy and regulation reserve markets under uncertainties. Energy, v. 121, p. 114$125,2017$.

ZAPATA RIVEROS, J. et al. Bidding strategies for virtual power plants considering CHPs and intermittent renewables. Energy Conversion and Management, v. 103, p. 408-418, 2015. 